\title{
PREDICTION FOR NEUTRINO-ELECTRON CROSS-SECTIONS IN WEINBERG'S MODEL OF WEAK INTERACTIONS
}

\author{
G. 't HOOFT \\ Institute for Theoretical Physics, University of Utrecht, Utrecht, the Netherlands
}

Received 27 October 1971

\begin{abstract}
Weinberg's theory of purely leptonic weak interactions can be tested in neutrino-electron scattering experiments. Cross-sections must be measured as a function of the energy of the recoil electron. If Weinberg's theory is correct, then the masses of the intermediate vector bosons can be derived from the measured effective coupling constants.
\end{abstract}

In an elegant way purely leptonic weak interactions can be cast in the form of a Yang-Mills theory, in which a local $\mathrm{SU}(2) \times \mathrm{U}(1)$ symmetry is broken spontaneously. Weinberg constructed such a model [1] and showed it to be consistent with all known data on leptons. Recently, furthermore, the author has shown that this model is renormalizable [2], which makes it an attractive alternative to many more complicated weak interaction theories. A problem arises if one tries to include hadrons: it is difficult to understand why the neutral intermediate $\mathrm{W}$-boson is not seen. Possibly the hadronic neutral current vanishes due to some mechanism.

Assuming Weinberg's model to be correct for leptons, we get very precise predictions for neutrino-electron elastic scattering. There is one arbitrary parameter, the weak coupling constant $g$. It is related to the mass of the intermediate vector bosons, $M_{ \pm}$and $M_{0}$, according to $g^{2}=4 \sqrt{2} G M_{ \pm}^{2} ; \quad M_{\mathrm{o}}^{2}=M_{ \pm}^{2}\left(1-e^{2} / g^{2}\right)^{-1}$ where $e^{2 / 4 \pi}=1 / 137$, and $G=1.02 \times 10^{-5} m_{\mathrm{p}}^{-2}$.

If the neutrino energy is small compared to the intermediate boson mass, the effective Lagrangian pertinent to $(e, \nu)$ scattering is

$$
\mathcal{L}=\frac{G}{\sqrt{2}} \bar{\nu} \gamma_{\mu}\left(1+\gamma_{5}\right) \nu \bar{e} \gamma_{\mu}\left(g \mathrm{~V}+g_{\mathrm{A}} \gamma_{5}\right) e
$$

It is obtained from the graphs depicted in fig. 1 (to the first of these we applied a Fierz-transformation). The contribution proportional to $e^{2} / g^{2}$ arises from an analogue of the vector dominance principle in strong interactions.

In Weinberg's model we have

$$
\text { for } \begin{aligned}
\left(\nu_{\mathrm{e}}, \mathrm{e}\right) \text { and }\left(\bar{\nu}_{\mathrm{e}}, \mathrm{e}\right) \text { scattering: } g_{\mathrm{V}} & =\frac{1}{2}+2 e^{2 / g^{2},} \\
g_{\mathrm{A}} & =\frac{1}{2} ;
\end{aligned}
$$

for $\left(\nu_{\mu}, \mathrm{e}\right)$ and $\left(\bar{\nu}_{\mu}, \mathrm{e}\right)$ scattering: $g_{\mathrm{V}}=-\frac{1}{2}+2 e^{2 / g^{2}}$,

$$
g_{\mathrm{A}}=-\frac{1}{2} \text {. }
$$

(In a theory with only charged intermediate bosons these numbers are 1, 1, 0,0 respectively).

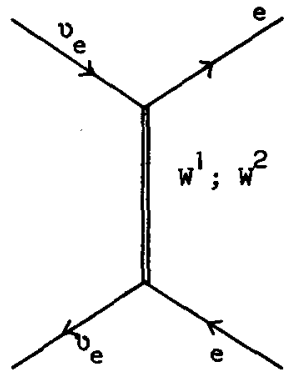

$g_{V}=g_{A}=1$

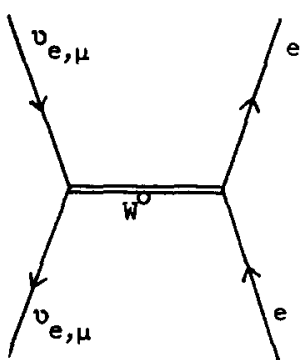

$\mathrm{B}_{\mathrm{V}}=\mathrm{B}_{\mathrm{A}}=-\frac{1}{2}$

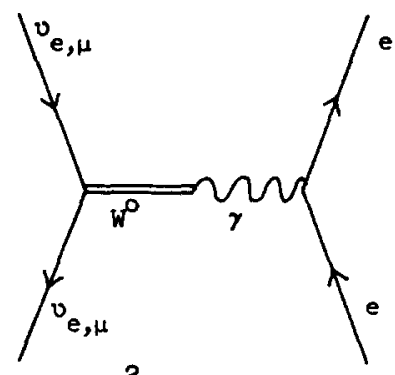

$B_{V}=\frac{2 e^{2}}{g} ; B_{A}=0$

Fig.1. 
The number $e^{2 / g^{2}}$ must fulfill the inequality

$0<e^{2}, g^{2}<1$.

(If $e^{2 / g^{2}} \leqslant 1 / 137$ then the perturbation expansion breaks down and the calculations are invalid, but there arises no inconsistency with what is known about muon decay etc.).

The quantities $g_{\mathrm{V}}$ and $g_{\mathbf{A}}$ can be measured if one knows the energy distribution of the neutrino beam, and measures the energy $E_{\mathrm{e}}$ of the recoil electron. Let the neutrino energy be $E_{\nu}$. The cross-section for neutrino-electron scattering is

$\frac{\mathrm{d} \sigma}{\mathrm{d} E_{\mathrm{e}}}=\frac{G^{2} m_{\mathbf{e}}}{2 \pi E_{\nu}^{2}} \times$

$\left[E_{\nu}^{2}\left(g_{\mathrm{V}}+g_{\mathrm{A}}\right)^{2}+\left(E_{\nu}-E_{\mathrm{e}}\right)^{2}\left(g_{\mathrm{V}}-g_{\mathrm{A}}\right)^{2}+m_{\mathrm{e}} E_{\mathrm{e}}\left(g_{\mathrm{A}}-g_{\mathrm{V}}^{2}\right)\right]$

The cross-section for antineutrino-electron scattering can be found from eq. (5) by replacing

$g_{\mathrm{A}}$ by $-g_{\mathrm{A}}$. Furthermore we have

$0<E_{\mathrm{e}}<E_{\nu} /\left(1+m_{\mathrm{e}} / 2 E_{\nu}\right)$.

If one can measure the angle $\theta$ between the recoil electron and the neutrino beams accurately, one finds $E_{\nu}$ directly: we have approximately

$\left(E_{\mathrm{e}, \nu} \gg m_{\mathrm{e}}\right)$ :

$E_{\nu} \approx \frac{2 E_{\mathrm{e}} m_{\mathrm{e}}}{2 m_{\mathrm{e}}-\theta^{2} E_{\mathrm{e}}}$, and $\theta$ lies in the range

$\theta \sim \sqrt{\frac{m_{\mathrm{e}}}{E_{\mathrm{e}}}}$.

We conclude that the proposed experiment could disprove or lend support to the theory. Besides, if Weinberg's theory is correct, then the intermediate boson mass can be found from the number $e^{2} / g^{2}$, following eq. (1).

The theory predicts (cf eqs. (1) and (4))

$$
M_{ \pm}>37 \mathrm{GeV}, \quad \quad M_{\mathrm{o}} \geqslant 74 \mathrm{GeV} \text {. }
$$

If $g_{\mathrm{V}}$ in $\left(\nu_{\mu} \mathrm{e}\right)$ scattering can be determined with at least $20 \%$ accuracy then the masses in (9) can be derived if they are lower than $\sim 150 \mathrm{GeV}$.

The author wishes to thank prof. M. Veltman for stimulating discussions and prof. S. Weinberg who brought this model to his notice.

\section{References}

[1] S. Weinberg, Phys. Rev. Letters 19 (1967) 1264.

[2] G. 't Hooft, Nucl. Physies, B35 (1971) 167. Anomalies due to parity non-conservation give rise to complications in the renormalization procedure, but do not make renormalization impossible. 